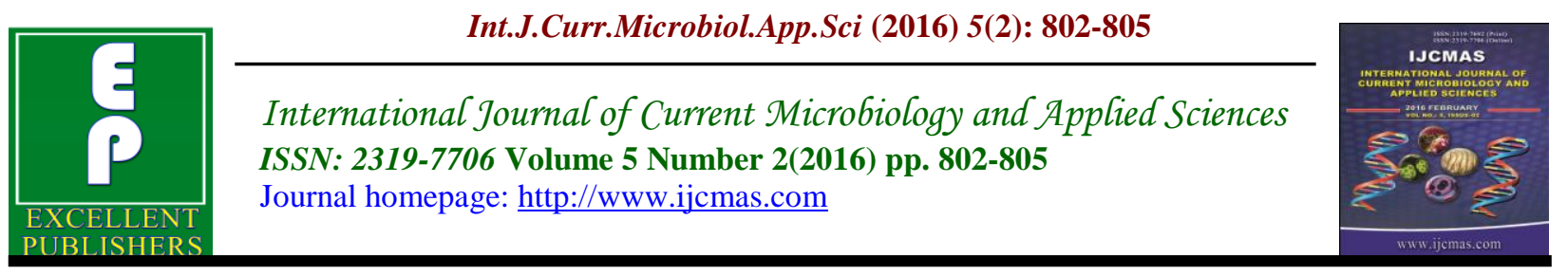

Original Research Article

doi: http://dx.doi.org/10.20546/ijcmas.2016.502.091

\title{
A comparative analysis of peripheral blood smear and rapid diagnostic test for diagnosing Malaria
}

\author{
Gunjan Dutta*, Kiran Yadav and Jugal Kishor Aggarwal \\ Department of Microbiology, Saraswathi Institute of Medical Sciences, Hapur (U.P), India \\ *Corresponding author
}

\begin{tabular}{|c|c|}
\hline & A B S T R A C T \\
\hline $\begin{array}{l}\text { Ke y w o r d s } \\
\text { Peripheral blood } \\
\text { smear, } \\
\text { Pan Malaria } \\
\text { Antigen Card } \\
\text { test. }\end{array}$ & \multirow{3}{*}{$\begin{array}{l}\text { Rapid detection of malarial parasites and early treatment of infection are the } \\
\text { most important goals of disease management. Blood sample was collected } \\
\text { from total number of } 1200 \text { patients present with classical symptoms of } \\
\text { malaria. Peripheral blood smears were prepared and stained with } \\
\text { Leishman's stain. Antigen detection tests were done using commercially } \\
\text { available kits. Out of } 1200 \text { patients } 202 \text { cases were positive by peripheral } \\
\text { smear and } 205 \text { were positive by card test. Pan Malaria antigen card test had } \\
\text { a sensitivity and specificity of } 100 \% \text { and } 99.70 \% \text { respectively. Peripheral } \\
\text { blood smear still appear superior for species identification but the RDTs are } \\
\text { easy to use, reliable and simple to interpret, therefore the test can be used as } \\
\text { an epidemiological tool for the rapid screening of malaria or where the } \\
\text { facilities are not available. }\end{array}$} \\
\hline Article Info & \\
\hline $\begin{array}{l}\text { Accepted: } \\
28 \text { January } 2016 \\
\text { Available Online: } \\
\text { 10, February } 2016\end{array}$ & \\
\hline
\end{tabular}

\section{Introduction}

Malaria is a mosquito borne infectious disease of humans and other animals caused by parasitic protozoan's belonging to genus Plasmodium(Malaria et al, 2014). The disease is transmitted by the bite of infected female Anopheles mosquito. If not appropriately treated people may have recurrences of the disease months later. The disease is widespread in the tropical and subtropical regions that exist in a broad band around the equator (Caraballo Het al, (2014)). Malaria possesses diagnostic challenge to the medical community worldwide.
It is a serious, sometimes fatal, parasitic disease posing a major public health problem in India. It is estimated that there are more than 50 million cases and 1.1-2.2 million deaths occur due to malaria every year (Shivlal et al, 1999;66:547-54). It continues to be one of the major health burden in developing countries. The earliest symptoms of malaria are very non-specific and variable which posess difficulty in clinical diagnosis but the treatment has to be started immediately in order to avoid complications (Sarita et al, 2012 ). 
Conventional peripheral blood smear examination for demonstration of malarial parasites remains the gold standard for diagnosing malaria. However this technique is time consuming and requires skilled personnel. Several newer diagnostic techniques have been designed to replace the conventional microscopic methods. Therefore the present study was done to compare the peripheral blood smear examination with pan-malaria antigen card test which detects the presence of lactate dehydrogenase of Plasmodium species.

\section{Materials and Methods}

The study was conducted during April 2014March 2015. The study group comprised of 1200 patients presenting with fever, chills, rigor and other suggestive symptoms of malaria attending various outpatient and inpatient departments of Saraswathi Institute of Medical Sciences(SIMS) hospital. Peripheral blood smears were prepared and stained with Leishman's stain and examined for oil immersion fields for malarial parasites by light microscopy (K.D. Chatterjee et al, 1980).

All the samples were subjected to rapid visual antigen card test (Company MAL CARD - J. Mitra) according to the manufacturer's instructions. The panmalaria antigen card test is a immunochromatographic card test for the qualitative detection of $\mathrm{pLDH}$ (lactate dehydrogenase) of all the four Plasmodium species (P.falciparum, P.vivax, P.malariae,
P.ovale) in human whole blood. The results were read in 5-20 minute. An interpretation of result was done as: Negative, when only one red line appears (control line) and positive, when two red lines appear (Control and Pan lines).

Results of peripheral blood smear and antigen card test were compared in all patients for their sensitivity and specificity

\section{Result and Discussion}

A total of 1200 samples were tested for malarial parasites. The blood film result indicated that 202(16.83\%) cases were infected with malaria and 998(83.16\%) cases were negative. Of these 184(91.08\%) cases were positive for P.vivax and 16 (7.92\%) cases were of P.falciparum.

Pan malaria antigen card test could detect 205 positive cases of malaria as shown in (Table-1). In the present study we evaluated the use of pan-malaria antigen card test for diagnosis of malaria, here we observed 3 cases of malaria were positive by card test and negative by blood smear examination.

Rapid detection and effective treatment is a prerequisite for reducing morbidity and mortality due to malaria. Peripheral blood smear study is simple, least expensive, labor intensive, time consuming and therefore delay in diagnosis. Leishman's or Giemsa stained blood smears are considered to be the "Gold standard" in diagnosis.

Table.1 Comparison of Leishman Stained Blood Film with Antigen Card Test

\begin{tabular}{|l|l|l|}
\hline Result & Blood Smear & Antigen Card test \\
\hline Positive & 202 & 205 \\
\hline Negative & 998 & 995 \\
\hline Total & 1200 & 1200 \\
\hline
\end{tabular}

"sensitivity of card test - 100\%, specificity-99.7\%,

"positive predictive value(PPV)-98.53 and negative predictive value(NPV)- 100\% 
However the interpretation of blood smear depends upon the good quality of microscope, staining technique with which blood film is prepared and also the concentration and motivation of skilled microscopist (M.A.Dowling et al, 34(1966), 249-267 and D. Payne, ,66(1988),621-626).

In the present study we observed $100 \%$ sensitivity, $99.7 \%$ specificity, $98.53 \%$ positive predictive value, and $100 \%$ negative predictive value in antigen card test comparing with the peripheral blood smear. Leishman stained thin blood smear detects malarial parasites only when there are 50 parasites/ml of blood (M.J.W. Pinto et al, 19(2001), 219-221). Similarly some authors also observed same findings (Sarita et al, 2012 and C.J. Palmer et al, 36(1998),203206).

However, in the present study we compared thin smear with antigen card test, 202 cases were positive by both the methods, while thin smear missed to rule out 03 cases which was positive by antigen card test (Table 1), which is comparatively similar to the study done by( Iqbal et al,68(5) (2003, 624-628).

However, stained smear is useful to keep a permanent record of the smear, its low cost and species identification without much difficulty in most of the cases. Newer techniques like Antigen detection assays are rapid, simple and easy to interpret but it should not be forgotten that a blood film can provide more information than card test and do not require any expertise.

To conclude our evaluation shows that MalCard antigen test is simple, reliable and rapid test for the diagnosis of malaria with some limitations like high cost, inability of quantification of parasite and differentiation between the Plasmodium species. Even then, the test can be a promising alternative to microscopy in places where the facility for microscopy are poor. Therefore, it is reasonable to consider future use of rapid card tests as an epidemiological tool for the rapid screening of malaria.

\section{References}

Caraballo H(2014) "Emergency department management of mosquito borne illness: Malaria, dengue and west nile virus". Emergency Medicine Practice 16(5).

Chatterjee, K.D., Examination of Blood for Parasites, In: parasitology (12 $2^{\text {th }}$ ed.), Calcutta, 1980

Dowling, M.A., and G.T. Shute, A comparative study of thick and thin blood films in diagnosis of scanty malaria parasitemia, Bull World Health Organ.,34(1966), 249-267.

health care level, Bull World Health Organ.,66(1988),621-626.

Iqbal, J. A. Muneera and N.A. Khalid et al, Performance of the optimal test for malaria diagnosis among suspected malaria at the rural health centers, American $\mathbf{J}$ of Tropical Medical Hygiene), 68(5) (2003, 624-628.

Malaria Fact Sheet N "94". WHO. March 2014. Retrieved 28 August 2014.

Palmer, C.J., J.F. Lindo, W.I. Klaskala, J.A.Quesada, R. Kaminsky, M.K. Banum et al, Evaluation of optimal test for rapid diagnosis of plasmodium vivaxand plasmodium falciparum malaria, J Clin Microbial., 36(1998),203-206.

Payne, D., Use and Limitations of light microscopy for diagnosing malaria at primary

Pinto, M.J.W., S.R. Rodrigues, R. Desouza and M.P. Verenkar, Usefulness of quantitative buffy coat blood parasite detection system in diagnosis of 
malaria, Indial Journal Medical Microbiology, 19(2001), 219-221.

Sarita Yadav, Madhu Sharma, Aparna and Uma C. Comparative Evaluation of Pan-Malaria Antigen Card Test and Blood Smear for Diagnosing Malaria, International $\mathrm{J}$ of Life
Sciences Biotechnology and Pharma Research.

Shivlal, Dhillon GP, Aggarwal CS epidemiology and Control of Malaria. Indian J Pediatr. 1999; 66:547-54.

\section{How to cite this article:}

Gunjan Dutta, Kiran Yadav and Jugal Kishor Aggarwa. 2016. A comparative analysis of peripheral blood smear and rapid diagnostic test for diagnosing Malaria. Int.J.Curr.Microbiol.App.Sci.5(2): 802-805. doi: http://dx.doi.org/10.20546/ijcmas.2016.502.091 